\title{
LOS ADVERBIOS EN -MENTE EN LAS PROPOSICIONES PROYECTORAS EN LA OBRA DE BENITO PÉREZ GALDÓS: UN ENFOQUE DE CORPUS
}

\author{
GuAdALUPE NiETO CABALLERO \\ Universidad de Extremadura
}

\section{Resumen}

En este artículo analizamos el uso que Benito Pérez Galdós hace de los adverbios acabados en -mente en su producción narrativa. El trabajo se centra en aquellos ejemplos utilizados en proposiciones proyectoras que introducen estilo directo. Para localizar los ejemplos, hemos empleado una metodología de corpus. En primer lugar, se ofrece una comparación del número de ejemplos identificados en el corpus galdosiano con aquellos localizados en un corpus de referencia de autores españoles decimonónicos. Una vez calibrada la frecuencia de uso en ambos corpus, analizamos el uso que Galdós hace de este elemento. El estudio se centra en tres patrones funcionales relacionados con la voz, el plano mental y el lenguaje gestual de los personajes, respectivamente. Como se verá, la profusión en el uso de adverbios por parte de Galdós se materializa en funciones estilísticamente relevantes, como la caracterización o la creación de universos ficticios que el autor nos plantea.

Palabras clave: Galdós, adverbios -mente, representación del discurso, enfoque de corpus.

\section{A CORPUS-BASED ANALYSIS OF ADVERBS ENDING IN -MENTE IN PROJECTING CLAUSES IN BENITO PÉREZ GALDÓS'S FICTIONAL NARRATIVES}

\begin{abstract}
In this article, we analyse Benito Pérez Galdós's use of adverbs ending in -mente in his fictional narratives. The study focuses on those examples identified in projecting clauses that introduce stretches of direct speech. In order to search for the examples under analysis, a corpus-stylistic approach was used. First, we carried out a comparison of the examples identified in a corpus of Galdós's novels against the backdrop of a reference
\end{abstract}


corpus made up of nineteenth-century Spanish fictional narratives. Then, we analysed the examples identified across Galdós's novels, focusing on three self-contained functional patterns: characters' voices, their psychological dimension and their body language. As will be shown, Galdós's extensive use of adverbs ending in -mente results in stylistically significant functions, such as characterisation and the construction of fictional universes.

Keywords: Galdós, adverbs ending in -mente, discourse presentation, corpus approach.

\section{INTRODUCCIÓN}

En este artículo se presenta un análisis de corpus sobre los adverbios acabados en -mente en las novelas de Benito Pérez Galdós. En concreto, se analiza el uso que Galdós hace de estos adverbios en proposiciones proyectoras $^{1}$ en el marco de la estrategia de representación del discurso de estilo directo, como se muestra en el ejemplo (1). El estilo directo es la estrategia de representación del discurso más empleada por Galdós (Nieto Caballero, 2018: 381), que le permitía perfilar personajes con un alto grado de individualización gracias a sus características voces. Como se verá, el uso que Galdós hace de adverbios en las proposiciones que introducen estilo directo no solo ayuda a compensar aspectos relacionados con la articulación del discurso que no pueden ser plasmados en la proposición proyectada, sino que mediante el uso de adverbios Galdós también incide en aspectos como el plano mental de los personajes o en interpretaciones sobre su lenguaje gestual. En conjunto, estos aspectos adquieren un importante valor estilístico, que puede contribuir a la caracterización de los personajes o a definir el universo ficticio que el autor nos plantea.

(1) -iOtro! Allá en lo profundo los condenados ordinarios no han de querer habitar con los renegados y traidores — dijo el hombre decrépito, silabeando enérgicamente con sus gruesos labios-. Los renegados venden a sus hermanos, entregan la patria al enemigo

\footnotetext{
${ }^{1}$ Siguiendo la nomenclatura propia de la gramática sistémica funcional, en este trabajo se utilizan los conceptos de proposición proyectora y proposición proyectada (Halliday, 2004: 445) para referirse a la voz del narrador y a las palabras de los personajes respectivamente.
} 
para que este la despoje y la deshonre a su antojo extirpando en ella la fe religiosa [...] (El equipaje del rey José, cap. 25)².

Para llevar a cabo el análisis, hemos empleado una metodología de corpus. Aunque este enfoque no resulta novedoso en los estudios literarios en general ${ }^{3}$, sí que lo es en los estudios de textos literarios en lengua española. El artículo podría englobarse bajo la disciplina de estilística de corpus (McIntyre y Walker, 2019), un enfoque que aplica «metodologías de corpus para el análisis de textos literarios, otorgando un énfasis particular a la relación entre la descripción lingüística y la apreciación literaria» (Mahlberg, 2014: 378) ${ }^{4}$. Gracias a su capacidad para procesar grandes cantidades de texto, las metodologías de corpus permiten detectar "patrones de los que nosotros como lectores no somos conscientes, a pesar de que dichos patrones podrían contribuir a los efectos que percibi-

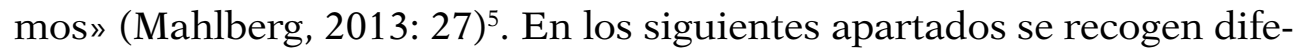
rentes usos y ejemplos de los adverbios en -mente, que se asocian, por lo general, con la voz de los personajes. Además de singularizar a los personajes de esta forma, se aprecian diferentes matices que contribuyen a caracterizarlos. En esta línea, el artículo servirá también para ilustrar el potencial de los estudios de corpus en el análisis de textos en nuestra lengua, un área todavía escasa de análisis que adopten este tipo de enfoques.

${ }^{2}$ Todos los ejemplos citados en el artículo han sido extraídos de una versión electrónica de los textos (véase apartado 3). Por lo tanto, en lugar de la paginación se ofrece como referencia el capítulo en el que aparecen.

${ }^{3}$ Dentro de la literatura anglosajona, por ejemplo, las obras de figuras de referencia como Joseph Conrad (Stubbs, 2005), Virginia Woolf (Balossi, 2014), William Shakespeare (Culpeper, 2009), Jane Austen (Fischer-Starcke, 2010) o Charles Dickens (Mahlberg, 2013; Ruano San Segundo, 2016), entre otras, han sido analizadas utilizando enfoques de corpus.

${ }^{4}$ La traducción es nuestra. La cita original es: "corpus methods to the analysis literary texts, giving particular emphasis to the relationship between linguistic description and literary appreciation».

${ }^{5}$ La traducción es nuestra. La cita original es: «patterns that we as readers may not be aware of, although such patterns might still contribute to the effects we perceive». 


\section{ADVERBIOS EN -MENTE}

Sintetizar la literatura científica en torno a los estudios sobre los adverbios en general en unas líneas es poco menos que imposible. Podríamos decir que esto se debe al "carácter negativo" (Domínguez de Rodríguez-Pasqués, 1970: 293) de este elemento, que ha llevado incluso a algunos a afirmar que «es adverbio lo que no es sustantivo, ni adjetivo, ni verbo ni relacionante» (Domínguez de Rodríguez-Pasqués, 1970: 293). Lázaro Carreter (1953: 24) se refería a esta categoría como «receptáculo universal». Esta «capacidad abarcadora» (García-Page, 1993: 311) se ha materializado en «el desdibujamiento de los límites de tal categoría y su consecuente confusión con otras clases de palabras» (García-Page, 1993: 311). En efecto, la heterogeneidad de los adverbios (morfológica, sintáctica y semántica) se ha consolidado como el aspecto más conflictivo en los trabajos de los gramáticos sobre esta clase de palabras, pues ha provocado dificultades a la hora de establecer límites, ofrecer clasificaciones y explicar su funcionamiento (Hernández Alonso, 1974; Carbonero, 1978; Hallebeek, 1985; Moreno de Alba, 1988; Vera Luján, 1979, entre otros) ${ }^{6}$.

Si estrechamos el foco de atención y nos centramos en los adverbios con el morfema -mente, el problema, lejos de desaparecer, despierta nuevos conflictos. García-Page (1993: 312), por ejemplo, critica el uso de un morfema como criterio de clasificación "para pretender dar cuenta de un grupo de signos que, aun presentando una configuración formal aparentemente idéntica, pueden llegar a corresponderse con valores funcionales muy diversos». Varios autores han tratado este problema (Egea, 1979; García-Page, 1991). Sin embargo, como el propio García-Page (1993: 312) reconoce, esta característica «ha propiciado que sea aislado del resto de los elementos adverbiales y se estudie aparte como un grupo muy particular». Eso es precisamente lo que hacemos en este artículo. Para ello, por supuesto, partimos del antedicho carácter heterogéneo del adverbio como clase de palabra en general y de los adverbios en -mente en particular. Un adverbio en -mente puede modificar la significación de un verbo, adjetivo, otro adverbio, un sustantivo e incluso una frase entera (Alarcos Llorach, 1969: 312). Aquí no nos centramos en su relación con ningún tipo de palaba en concreto, sino que hemos acotado su aparición

\footnotetext{
${ }^{6}$ Para una lista más extensa, véase García-Page (1993: 312).
} 
en una construcción concreta - proposiciones proyectoras que introducen estilo directo- $-\mathrm{y}$ hemos analizado todos los ejemplos sin menoscabo de los elementos con los que se asocia.

El hecho de centrarnos en una construcción y no en la asociación de los adverbios con otros elementos tiene que ver con la naturaleza de la lengua literaria. Al contrario de lo que ocurre en otros ámbitos como la lengua hablada, donde se advierte una "parquedad» y un «repertorio reducido y trillado» de estas formas (Casares, 1992: 157), en el texto literario se observa «mayor libertad y originalidad de creación» (Domínguez de Rodríguez-Pasqués, 1970: 295), por lo que es más probable encontrar usos menos idiomáticos e incluso inéditos de estas construcciones. Efectivamente, las estrictas reglas de selección «que, en la lengua estándar, gobiernan el procedimiento de formación de palabras mediante el sufijo -mente son, sin embargo, transgredidas con cierta frecuencia en determinados registros idiomáticos» (García-Page, 1993: 313) como el literario. De hecho, no es extraño encontrar trabajos de gramáticos apoyándose en ejemplos extraídos de textos literarios. Domínguez de Rodríguez-Pasqués (1970: 298), por ejemplo, utiliza ejemplos de Cortázar (apoyarse pelirrojamente contra una puerta, ser dostoievskianamente asqueroso, sonreír homéricamente entre lágrimas) para referirse a algunos usos inéditos de corte humorístico. Huelga decir que el interés de este artículo no reside en localizar tales usos literarios, sino en identificar patrones dentro de una construcción concreta que tiene que ver con la enunciación. Como se verá, aunque muchos de estos adverbios actúan como modalizadores en actos de enunciación como los que aquí se analizan (Lakokff, 1970; Kovacci, 1980; Fuentes Rodríguez, 1987), otros desempeñan otras funciones igualmente relevantes. Por lo tanto, y teniendo en cuenta la distinta gramática y comportamientos de cada adverbio (García-Page, 1993: 322), hemos preferido centrarnos en patrones funcionales.

Más allá de estas cuestiones relacionadas con el enfoque que hemos adoptado, lo que parece claro es que Galdós era bien consciente del potencial de estos adverbios. En su reflexión sobre la lengua en La conjuración de las palabras, de 1868, se refería a ellos del siguiente modo:

Tras estos [los verbos] venían los Adverbios, que tenían cataduras de pinches de cocina; como que su oficio era prepararles la comida a los Verbos y servirles en todo. Es fama que eran parientes de los 
Adjetivos, como lo acreditaban viejísimos pergaminos genealógicos, y aun había Adjetivos que desempeñaban en comisión la plaza de Adverbios, para lo cual bastaba ponerles una cola o falda que, decía: mente.

En este artículo veremos cómo el autor canario emplea con profusión estos adverbios en momentos de representación de discurso con diversos fines, como la caracterización o la construcción de los universos ficticios que nos plantea.

\section{CORPUS DE ESTUDIO Y CORPUS DE REFERENCIA}

El análisis se ha llevado a cabo utilizando dos corpus: uno de estudio con la obra de Galdós (en adelante, CorBPG) y otro de referencia con novelas del siglo XIX (en adelante, CorXIX). Este último se ha utilizado por una cuestión de comparación, con el fin de calibrar la relevancia de los resultados obtenidos en la producción de Galdós. Los textos que forman ambos corpus de estudio han sido descargados del repositorio digital de la Biblioteca Virtual Miguel de Cervantes (en línea: $<$ http://www.cervantesvirtual.com/>). Para llevar a cabo el análisis, hemos seleccionado todas las novelas del autor canario, con una extensión de más de seis millones de palabras, como se muestra en el apéndice 1 . La narrativa breve no ha sido incluida en el corpus, por no tratarse de novelas propiamente dichas. Tampoco ha sido incluida su producción teatral, por no pertenecer al género narrativo.

El corpus de referencia de novela realista, por su parte, está formado por ochenta novelas de ocho autores diferentes: Pedro Antonio de Alarcón, Vicente Blasco Ibáñez, Leopoldo Alas "Clarín», Luis Coloma, Armando Palacio Valdés, Emilia Pardo Bazán, José María de Pereda y Juan Valera. Las ochenta novelas tienen una extensión similar a CorBPG, con 6.188.478 palabras. Los títulos incluidos en CorXIX se muestran en el apéndice 2. Para construir nuestro corpus de referencia, hemos seguido las nociones de representatividad y pertinencia de los textos propias de los estudios de lingüística de corpus en general. Por un lado, la representatividad tiene que ver con que la muestra de referencia sea lo suficientemente extensa como para que admita una comparación con el objeto de estudio (en nuestro caso, la producción novelística de Galdós). Por ello 
se ha escogido un corpus de aproximadamente la misma extensión que el corpus de estudio, de tal suerte que resulte una muestra lo suficientemente fiable desde un punto de vista cuantitativo. En cuanto a la pertinencia de los textos, todos pertenecen al género narrativo y al mismo movimiento literario que los textos del corpus de estudio, como ya se ha comentado. Conviene incidir en la presencia desigual de cada autor en el corpus, debida al distinto grado de prolijidad de los distintos novelistas. En otras palabras, autores con una producción más reducida, como «Clarín», gozan de menos peso en el corpus de referencia que otros más prolijos, como Pardo Bazán. En cualquier caso, CorXIX constituye una muestra de la literatura realista española lo suficientemente sólida como para poder realizar una comparación cabal de los resultados obtenidos en el corpus galdosiano y establecer su representatividad antes de analizarlos.

\section{MEtodología y RESUltados}

Para realizar el análisis, hemos empleado el software de concordancias WordSmith Tools (Scott, 2013). Para calibrar el uso que Galdós hace de los adverbios en -mente en momentos de proyección del discurso, primero debemos localizar estos adverbios en toda la producción de Galdós (CorBPG), así como en CorXIX, con el fin de realizar una comparación cabal. Para ello, basta con generar una lista de palabras de cada corpus y seleccionar aquellas con terminación en -mente. A continuación, se descartan los sustantivos (clemente, demente), los adjetivos (vehemente) y formas verbales (comente o comenté, ya que el programa no distingue tildes y localiza indistintamente -mente y -menté) que no son adverbios. Tras realizar este cribado, en nuestra lista de palabras permanecen únicamente los adverbios en -mente. En CorBPG se han localizado 20.999 ejemplos de 1.066 adverbios diferentes, mientras que en CorXIX hemos identificado 23.031 ejemplos de 1.270 adverbios. Para comprender mejor estas cifras, debemos normalizar las frecuencias de uso, como se muestra en la tabla 1. Como se puede advertir, Galdós utiliza 3,26 adverbios por cada mil palabras de texto, por los 3,73 adverbios del resto de autores 
decimonónicos ${ }^{7}$. Asimismo, la ratio type-token muestra una mayor riqueza léxica en CorXIX, pues el uso medio de cada adverbio localizado tiene una frecuencia de uso menor: 18,13 ejemplos de media en el caso de CorXIX por los 19,69 de CorBPG. Así, aunque en el trazo grueso podríamos decir que el uso es similar, estos datos revelan que Galdós se sitúa en peldaño por debajo tanto en frecuencia como en variedad de uso con respecto a los novelistas de su tiempo.

\begin{tabular}{|c|c|c|c|c|}
\hline CORPUS & TYPES & TOKENS & $\begin{array}{c}\text { FRECUENCIA NORMALIZADA } \\
\text { (USO POR CADA MIL PALABRAS) }\end{array}$ & $\begin{array}{c}\text { RATIO TYPE- } \\
\text { TOKEN }\end{array}$ \\
\hline CORBPG & 1066 & 20999 & 3,26 & 19,69 \\
\hline CORXIX & 1270 & 23031 & 3,73 & 18,13 \\
\hline
\end{tabular}

TABLA 1. Frecuencia de uso de adverbios en -mente en CorBPG y CorXIX

Esta información resulta fundamental para calibrar el uso que Galdós hace de estos adverbios en proposiciones proyectoras que introducen estilo directo, como se explica más adelante. Para medir este uso, hemos hecho una concordancia avanzada de la forma dijo, variante más frecuente del verbo de habla por excelencia en Galdós y en la novela realista española para introducir estilo directo (Nieto Caballero, 2018: 381). En concreto, hemos hecho una concordancia de la forma dijo combinada con cualquier palabra acabada en -mente que aparezca en un rango de diez palabras a la derecha (R10) de la matriz (dijo), como se muestra en la imagen 1. Aunque pueda parecer un rango demasiado extenso, el margen de diez palabras no resulta desatinado. Como se puede advertir en el ejemplo (2), en las proposiciones proyectoras se incluye habitualmente información de distinto tipo (entre la que se encuentran los adverbios en -mente) que hace que sean más extensas de lo que en ocasiones cabría esperar.

(2) -Nada de eso hace al caso — dijo Regato como hombre afanado que se decide a marchar derechamente hacia su objeto-. Usted creerá tal vez que yo no correspondería a su buena voluntad con

${ }^{7}$ Esta frecuencia de uso es similar a la detectada por Domínguez de Rodríguez-Pasqués (1970: 302) en su trabajo (3,9 adverbios por cada mil palabras de texto), si bien su muestra es más reducida. 
otra buena voluntad, a su beneficio con otro beneficio (El Grande Oriente, cap. 24).

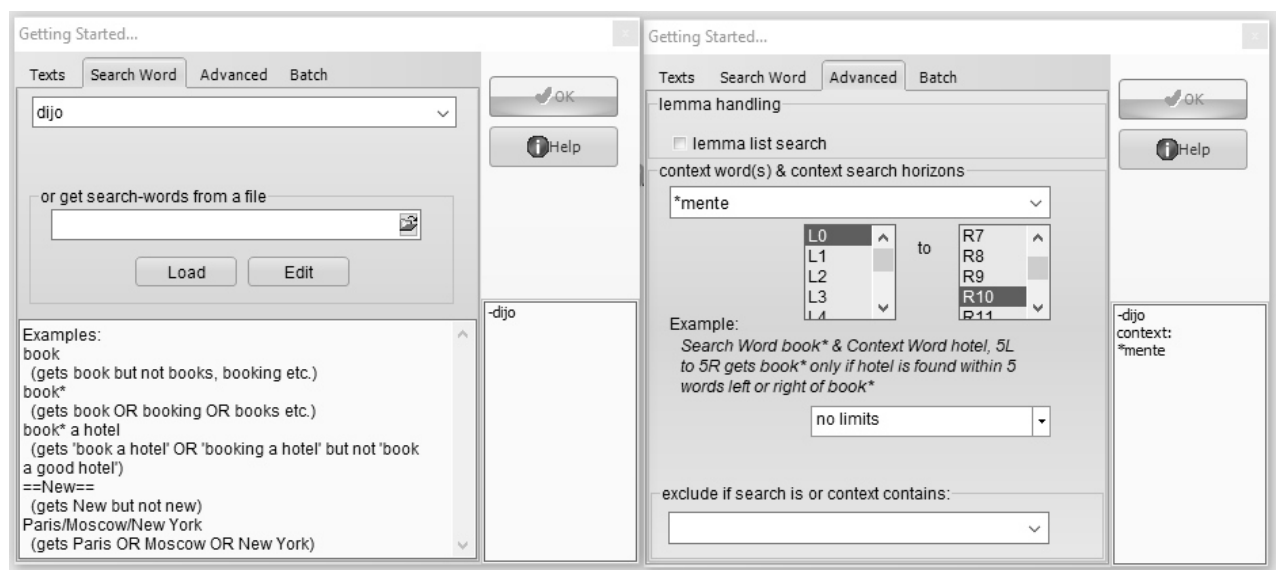

ImAGEN 1. Captura de pantalla de WordSmith Tools de una concordancia avanzada con dijo y -mente

Esta concordancia identifica 985 ejemplos en CorBPG y 499 en CorXIX. Sin embargo, esta lista de ejemplos ha de filtrarse para eliminar aquellos casos en los que no estamos interesados. Por ejemplo, cuando el adverbio forma parte de la proposición proyectada y no de la proyectora, como se muestra en (3).

(3) — Cuánto me alegro de verle a usted, amiguito! —dijo D. Buenaventura-. Precisamente necesitaba hablar a usted para ponerle sobre aviso [...] (El audaz: historia de un radical de antaño, cap. 13).

Una vez filtrada, en la concordancia de CorBPG permanecen un total de 646 ejemplos que se ajustan al patrón de estudio, mientras que en CorXIX permanecen 369. Como se puede observar en la tabla 2, estas cifras revelan una frecuencia de uso mucho mayor por parte de Galdós de la fórmula «dijo + adverbio en -mente». En concreto, en CorBPG 3,07 de cada cien adverbios se localizan en este patrón, mientras que en el CorXIX solo 1,60 de cada cien adverbios en -mente se emplean en estas circunstancias. En otras palabras, a pesar de que hay más uso en general de adverbios en CorXIX que en CorBPG (tabla 1), en la narrativa de Galdós se advierte un uso casi el doble de frecuente que en la novela del XIX (tabla 2). Sin duda, este dato revela un hábito galdosiano por oposición 
al resto de autores que forman CorXIX. Como se analiza a continuación, en este hábito se advierten además funciones estilísticamente relevantes.

\begin{tabular}{|c|c|c|c|}
\hline CORPUS & TOKENS & EJEMPLOS LOCALIZADOS & $\begin{array}{c}\text { FRECUENCIA NORMALIZADA } \\
\text { (USO POR CADA CIEN PALABRAS) }\end{array}$ \\
\hline CORBPG & 20999 & 646 & 3,07 \\
\hline CORXIX & 23031 & 369 & 1,60 \\
\hline
\end{tabular}

TABLA 2. Frecuencia de «dijo + adverbio en -mente» obtenidos en CorBPG y CorXIX

\section{ANÁLISIS}

Cabe destacar, antes de analizar los ejemplos, que la utilización de adverbios en -mente en proposiciones proyectoras no es, en ningún caso, una extrañeza estilística. Como se comentaba en la introducción, este es un recurso frecuente para compensar aspectos que no se incluyen en el acto de habla que se proyecta cuando se recurre al estilo directo. La particularidad de Galdós está en la frecuencia que acaba de apuntarse en el apartado anterior con respecto al resto de autores realistas, así como en las funciones que tiene en sus novelas.

Sobre el uso que Galdós hace de los adverbios localizados en proposiciones proyectoras, los 646 ejemplos localizados se dividen en 177 adverbios diferentes. Como se ha apuntado, todos siguen el mismo patrón: proposición proyectora que introduce el estilo directo mediante la forma dijo. Sin embargo, cabe destacar que los adverbios no se asocian necesariamente a dijo, sino también a otros elementos incluidos en la proposición proyectora, como se puede observar en terciando airosamente la capa en que se envolvía del ejemplo (4). De hecho, ni siquiera se asocian siempre a verbos. En ocasiones tienen que ver con sustantivos o adjetivos, como en hondamente preocupado en (5). Estos dos ejemplos, de hecho, revelan cómo el uso que Galdós hace de los verbos va más allá del plano discursivo. De los distintos aspectos identificados en los 646 ejemplos localizados nos ocupamos a continuación. 
(4) - La buena vida, amigo — dijo con petulancia, terciando airosamente la capa en que se envolvía-. Ya no estoy en las cocinas; he pasado a la montería del señor infante D. Antonio Pascual, donde no hay mucho que hacer y se divierte uno. [...](El 19 de Marzo y el 2 de Mayo, cap. 7).

(5) —iOh! — dijo Lantigua hondamente preocupado-. Es preciso ante todo redimirle a usted de esta horrible abominación pública, indigna de la cultura moderna (Gloria, cap. 11).

\subsection{Funciones}

Existen tres planos bien diferenciados en el uso que Galdós hace de los adverbios en -mente en las proposiciones proyectoras: el plano discursivo, relacionado directamente con el modo de articular discurso de los personajes (apartado 5.1.1); el plano mental, relacionado con la dimensión psicológica de los personajes (apartado 5.1.2); y el apartado del lenguaje gestual, relacionado con las acciones de los personajes que se describen al tiempo que se introduce su discurso (apartado 5.1.3). El uso que Galdós hace de los adverbios en estos tres planos puede ayudar a delinear a los personajes (su caracterización) e incluso a construir el universo ficticio que el autor nos plantea. Para ofrecer una visión lo más nítida posible de cada plano, el análisis de estos se ha dividido en tres subapartados.

\subsubsection{La voz de los personajes}

El de la voz de los personajes es sin duda el plano en el que encontramos más ejemplos. Hasta 473 (73,2\% de los ejemplos) tienen que ver con el modo de articular discurso. Como sostiene Caldas-Coulthard (1987: 165), cuando recurren al estilo directo y a formas de decir neutrales (en el caso del español, decir), los novelistas usan con frecuencia elementos que sirven para compensar la falta de matices del verbo de habla. CaldasCoulthard utiliza el ejemplo de los adverbios en -ly en inglés, que no son sino el equivalente a los adverbios en -mente que aquí se analizan. Estos adverbios pueden depender de dijo o de algún sintagma que lo acompañe. En Galdós encontramos ejemplos en ambos sentidos. En (6) se muestra un ejemplo que se asocia con el verbo de habla, mientras que en (7) el adverbio aparece dentro de una oración subordinada (suspirando tristemente). Además, estos adverbios pueden tener que ver con el modo de 
articulación (cuestiones relacionadas con la dicción) o con la actitud del personaje. De nuevo, (6) sería un buen ejemplo del primer caso y (7) del segundo.

(6) -Pues tendrás la bondad — dijo lentamente Amparo, registrando su bolsita y sacando un papel-, de ir a la botica, que está en esta misma calle, dos puertas más abajo... (Tormento, cap. 34).

(7) —No hay nada —dijo la muchacha suspirando tristemente-. Se ha concluido lo que tú trajiste la semana pasada, y hace dos días que la señora Sumta no me da la más mínima hora, porque parece que arriba faltan también las provisiones. ¿Nos traes algo esta noche? (Gerona, cap. 6).

Dada la frecuencia con la que Galdós recurre a adverbios en -mente para modelar el discurso de los personajes, no es extraño encontrar patrones que vayan más allá del plano meramente discursivo. Por ejemplo, la información que revelan los adverbios que se relacionan con la voz de los personajes puede ser importante en términos de caracterización a través de la evaluación del acto de habla que se representa. Como afirma Culpeper en su modelo sobre la caracterización en textos literarios, el modo en el que uno habla puede revelar información sobre el carácter de los personajes, pues hay «una relación sólida entre ciertas voces y ciertos tipos de personalidad»(Culpeper, 2001: 15) ${ }^{8}$. Esto es, no solo es importante lo que un personaje dice, sino cómo lo dice. Sirva el caso de Daniel Morton, en Gloria, como ejemplo. En los ejemplos (8) a (11) vemos cómo los adverbios localizados junto a la forma dijo contribuyen a definir la dimensión trágica del judío (Pattison, 1954: 93) a través de su forma de hablar, descrita siempre en términos similares: gravemente, sombríamente y lúgubremente.

(8) -Yo soy judío — dijo Morton gravemente (Gloria, cap. 10).

(9) -Las leyes del verdadero honor — dijo Morton gravemente-, son las leyes morales que emanan de la religión o de la filosofía. Fuera de esto, todo es convencional y falso (Gloria, cap. 11).

\footnotetext{
${ }^{8}$ La traducción es nuestra. La cita original es: «a strong relationship between certain voices and certain personality types».
} 
(10) —No, madre, esto no es morir — dijo Morton lúgubremente-. Quiero resucitar a esa pobre mujer que adoro [...](Gloria, cap. 27).

(11) —iLa religión! —dijo Morton sombríamente-. ¡Siempre el mismo fantasma pavoroso que nos persigue atormentándonos! [...] (Gloria, cap. 32).

Es cierto que, si los analizáramos individualmente, cada ejemplo parece simplemente contextualizar el acto de habla. En conjunto, sin embargo, la información que sistemáticamente recibimos como lectores a través de gravemente, sombríamente y lúgubremente contribuye a definir la caracterización de Morton a través de una suerte de efecto acumulativo (Montoro, 2012: 89) en el transcurso de su vida textual. Esta disposición, así como su valor caracterizador, entronca con enfoques propios de la estilística cognitiva (West, 2016) y con teorías sobre la percepción de los personajes de ficción, como el «mind-modelling» (Stockwell, 2009) $)^{9}$. Efectivamente, debido al elevado grado de atención que el lector dedica a comprender la dimensión mental de los personajes (Hakemulder, 2016), la repetición en el transcurso de la historia de la información sobre el modo de hablar de Morton proporcionada en sus intervenciones a través de los adverbios en -mente puede ayudar a definir la imagen que Galdós proyecta de él y que, como lectores, nos formamos en el acto de lectura de la novela. Este valor de los adverbios encaja, además, con el valor caracterizador de la información que Galdós incluye habitualmente en las proposiciones proyectoras comentado en estudios previos (Nieto Caballero, 2018: 385-88).

\subsubsection{Plano mental de los personajes}

Aunque de manera mucho menos frecuente, los adverbios también se relacionan a menudo con la dimensión psicológica de los personajes (49 ejemplos). Relacionado con ello existen dos aspectos destacados. Por un lado, las referencias al plano mental en momentos de representación discursiva se producen habitualmente en momentos en los que el personaje ofrece, de cara al mundo exterior, una imagen que no se corresponde con lo que verdaderamente siente. El contraste entre lo que el personaje dice

\footnotetext{
${ }^{9}$ En términos generales (esto es, más allá del ámbito literario que aquí nos ocupa), el mind-modelling puede definirse como el "proceso por el cual percibimos y entendemos mentes distintas a la nuestra» (Harrison, 2017: 30). La cita original es: "process by which we perceive and understand minds other than our own".
} 
(el estilo directo) y lo que de verdad siente (que el narrador describe en la proposición proyectora) se encuentra con frecuencia modalizado por adverbios, como se puede advertir en el uso de medianamente en los ejemplos (12) y (13). Aunque en el primer ejemplo el sentimiento que se oculta es de disgusto y en el segundo es de emoción, la lógica detrás de ambos ejemplos es similar. En ellos el narrador no solo nos informa de que hay una discrepancia entre el estado anímico del personaje y lo que dice, sino que sugiere (mediante el uso del adverbio) que esa discrepancia no se logra ocultar del todo ante el interlocutor, sino simplemente disimular.

(12) - Bueno es el cuadro, bueno - dijo el otro, ocultando medianamente su disgusto - . Cuando sea realidad avise usted... Me consolaré de mi tristeza viendo la alegría de los que con sus buenas acciones han merecido vivir en paz (Un faccioso más y algunos frailes menos, cap. 1).

(13) —Eso sí que no lo he de creer —dijo ella, afectando jovialidad para encubrir medianamente su emoción-. ¿Tan pronto?... No vengas ahora con palabrotas [...](Doña Perfecta, cap. 8).

Por otro lado, Galdós también se muestra mucho más determinante en la revelación de emociones de sus personajes mediante el uso de adverbios. Así, cuando el personaje no pretende ocultar cómo se siente, Galdós suele recurrir a adverbios con un valor intensificador: claramente, completamente, definitivamente, hondamente, enteramente, etc. En los ejemplos (14) y (15) se muestran casos de totalmente, enteramente y completamente. Como se puede advertir, estos ejemplos guardan además similitudes formales, pues suelen asociarse al adjetivo que revela el estado del personaje (totalmente aplacado, enteramente trastornada, completamente acobardado) para intensificar su significado.

(14) - Yo le suplico a usted, mi Sr. D. Romualdo — dijo Doña Francisca enteramente trastornada ya-, que no crea nada de eso [...] (Misericordia, cap. 33).

(15) — Bah... ya viene usted de malas — dijo Torquemada con fingido humor de bromas, y completamente acobardado-. ¿Y qué?, ¿no tengo más remedio que creer en la existencia de ese centro todo 
lleno de lumbre, y en los diablos, y en que todo ello debe durar eternidades? (Torquemada y San Pedro, cap. 2).

\subsubsection{Lenguaje gestual de los personajes}

En tercer lugar, los adverbios identificados en las proposiciones proyectoras también se emplean con frecuencia para describir casos de lenguaje gestual, un aspecto que aparece casi indisolublemente unido a la representación del discurso verbal en el género novelesco, sobre todo en estilo directo (Korte, 1997: 94 sigs.). Hasta 124 ejemplos se engloban en esta categoría. Estos ejemplos pueden dividirse a su vez en tres grupos: aquellos en los que se menciona alguna parte del cuerpo, aquellos que hacen referencia a las miradas de los personajes y los que se asocian con alguna acción que implica movimiento. Sobre las referencias a partes del cuerpo, se han identificado hasta treinta referencias explícitas a las siguientes partes del cuerpo: las manos, los hombros, los brazos, los labios, los pies, la cabeza, el ceño, el entrecejo, el pecho y la nariz de los personajes. Los adverbios juegan un papel clave en estos casos, pues ofrecen una interpretación sobre el valor que en realidad tiene el lenguaje gestual descrito. Esto es, mientras que las descripciones de lenguaje gestual sirven para contextualizar, el hecho de que aparezca acompañadas por un adverbio les otorga un componente interpretativo que nos ayuda a entender mejor el acto de habla al que acompañan. Sirvan como ejemplo los casos de cariñosamente y furiosamente que se muestran en (16) y (17). En ambos casos, el uso de los adverbios sirve para revelar la actitud de los personajes, lo que facilita la interpretación de sus palabras.

(16) —Sí, todo humo — dijo Fortunata, poniéndole cariñosamente la mano en el hombro-. No pienses y no temerás nada. Es la imaginación, nada más que la imaginación [...] (Fortunata y Jacinta, parte 4 , cap. 1).

(17) —Muy bien — dijo el presbítero restregándose furiosamente las manos-. Eso no podía faltar... Aparece la lógica en medio de este barullo romántico [...](Mendizábal, cap. 7).

Sobre las miradas hemos localizado 26 ejemplos, en donde los adverbios transforman nuevamente descripciones que en principio solo contextualizan en casos con un valor interpretativo estilísticamente signifi- 
cativo. En (18), Galdós se refiere a una mirada que proyecta matices positivos. Así, Miss Fly le dice a Gabriel lo valiente y generoso que es mirándole «fijamente al rostro». El adverbio fijamente es el que más se asocia a las miradas, con ocho usos. Además, las miradas también pueden jugar una función importante en la construcción del universo ficticio cuando se acompañan de adverbios. Tal es el caso de alternativamente, el segundo más frecuente, con tres ejemplos. En (19) podemos observar cómo el narrador controla la focalización a través de la mirada del personaje. Este uso que Galdós hace de adverbios como alternativamente con la mirada de los personajes sirve para reforzar el dominio del espacio visual y el movimiento como un recurso con el que «se prefigura el ritmo cinematográfico» de muchas de sus obras (Arroyo Díez, 2011: 366).

(18) -Sois valiente y generoso, sin duda — dijo mirándome fijamente al rostro-. Bien se conoce en vuestro semblante que lleváis en las venas la sangre de aquellos insignes caballeros que han sido asombro y envidia de Europa por espacio de muchos siglos ( La Batalla de los Arapiles, cap. 9).

(19) - Hace tiempo que tu buen padre me habló de un dulce proyecto que me agradaba en extremo, Carlos —dijo el viejo mirando alternativamente a su nieta y al joven guerrillero-. ¿Sabes lo que quiero decir? [...] (El equipaje del rey José, cap. 25).

De hecho, este ritmo cinematográfico también se observa en el uso de adverbios que se asocian con acciones que implican movimiento (68 en total). Como se puede advertir en (20), en las proposiciones proyectoras también se introducen descripciones relacionadas con qué está ocurriendo al tiempo que el personaje articula su discurso, lo que contribuye a definir el universo ficticio que el autor nos plantea. Estas descripciones se encuentran con frecuencia matizadas por un adverbio, que, como se puede advertir en el uso de nerviosamente en (20), pueden revelar información importante sobre el estado de un personaje.

(20) - Todo sea por Dios — dijo liando nerviosamente otro cigarriIlo-. Noble criatura, su juventud de usted ha sido muy triste; ha nacido usted en un páramo... (La desheredada, parte 1, cap. 1). 


\subsection{Aspectos formales}

Por último, aunque desde luego no por ello menos importante, en el plano formal también se advierten rasgos dignos de análisis. Formalmente, la proposición proyectora en la que se encuentra el adverbio aparece interrumpiendo la proposición proyectada, partiendo esta en dos. Este tipo de proposición proyectora que interrumpe el parlamento del personaje en la estrategia de estilo directo se denomina suspensión (suspension), y puede definirse como «una interrupción prolongada del discurso del personaje por parte del narrador» (Lambert, 1981: 6) ${ }^{10} .409 \mathrm{de}$ los 646 ejemplos localizados aparecen en suspensiones (un 63,1\%). De manera similar a Dickens, Galdós coloca proposiciones proyectoras suspendidas cuando desea concentrar información relacionada con la caracterización de un personaje (Mahlberg y Smith, 2012: 60). Como hemos visto a través del ejemplo de Morton en el apartado 5.1.1, en Galdós también observamos estos usos. Y, sin duda, lo más importante: las suspensiones son un lugar habitual para la descripción de lenguaje gestual. Como apuntan Mahlberg, Smith y Preston (2013: 40), «las suspensions pueden crear una impresión de simultaneidad entre el discurso y la información contextual descrita por el narrador, lo que a su vez puede sugerir similitudes con el desarrollo simultáneo del habla y el lenguaje corporal en la vida real ${ }^{11}$. En relación con ello, Galdós se apoya en diferentes recursos para construir estas suspensiones y ofrecer un efecto de sincronía entre las palabras del personaje y sus acciones. En concreto, cuando las suspensiones tienen que ver con el lenguaje gestual de los personajes, Galdós recurre frecuentemente a vocativos en la primera parte del parlamento del personaje ${ }^{12}$. Como se puede advertir en los ejemplos (21) y (22), Galdós parece recurrir a vocativos y exclamaciones como es-

${ }^{10}$ La traducción es nuestra. La cita original es: «a protracted interruption by the narrator of a character's speech».

${ }^{11}$ La traducción es nuestra. La cita original es: «suspensions can create an impression of simultaneity between the speech and the contextual information described by the narrator, which in turn can suggest similarities to the simultaneous occurrence of speech and body language in real life».

${ }^{12}$ Véase el estudio de Mahlberg, Smith y Preston (2013: 51-53) para comprender este recurso en literatura, centrado, de manera específica, en la obra de Dickens. 
trategia para poder suspender la proposición proyectora y crear la antedicha impresión de simultaneidad entre las palabras de los personajes y sus acciones.

(21) — Sr. D. Inocencio — dijo doña Perfecta, mirando alternativamente a su sobrino y a su amigo- creo que Vd. al juzgar a este chico, traspasa los límites de la benevolencia [...] (Doña Perfecta, cap. 7).

(22) - Caballero — dijo súbitamente deteniendo el paso-. Veo que os estáis burlando de mí (La batalla de los Arapiles, cap. 9).

\section{CONCLUSIONES}

Como se ha expuesto a lo largo de los distintos apartados, Galdós se sirve de adverbios acabados en -mente en las proposiciones proyectoras que introducen estilo directo. Como se ha visto, a pesar de que en general Galdós utiliza los adverbios en -mente de manera menos habitual que la nómina de autores realistas de CorXIX, lo cierto es que en las proposiciones proyectoras que introducen estilo directo se advierte un uso casi el doble de frecuente que en la novela del XIX. Esta frecuencia, además, cristaliza en funciones concretas, de las que se pueden observar en ejemplos a lo largo de toda su producción. La mayor parte de los ejemplos se asocian con la voz de los personajes. Además de compensar matices que no se pueden ofrecer de otra manera, en los adverbios también se advierten patrones que tienen que ver con la caracterización y que pueden contribuir, a través de la proyección de un modo de hablar concreto, a perfilar la imagen de un personaje en el transcurso de la historia.

La frecuencia de uso de este tipo de adverbios por parte de Galdós en las proposiciones proyectoras, así como su valor estilístico, sugieren nuevas vías de análisis en el estudio de las técnicas de representación del discurso del autor canario. A la luz de los resultados aquí expuestos, cabe pensar que, además de adverbios en -mente, Galdós podría recurrir a otros elementos con los que proyectar el discurso de sus personajes de forma más frecuente que el resto de novelistas decimonónicos. Esto se materializaría en un hábito estilístico típicamente galdosiano, que ven- 
dría a reforzar sus conocidos esfuerzos por dotar a sus personajes de hablas reconocibles y con un alto grado de matices. Esta es una vía que podría explorarse utilizando un enfoque de corpus como el adoptado en este trabajo, pues permitiría calibrar y comparar frecuencias de uso y realizar análisis sistemáticos de esos hábitos.

Precisamente es el enfoque de estilística de corpus adoptado en este trabajo el que nos ha permitido llevar a cabo nuestro análisis. Gracias a una metodología de corpus ha sido posible no solo calibrar el uso que Galdós hace de los adverbios (y compararlo con un corpus de referencia de autores de su época) sino analizar sistemáticamente funciones que tradicionalmente han pasado desapercibidas en la exégesis del novelista canario. En este sentido, consideramos que este artículo también puede contribuir a ilustrar el potencial de las metodologías de corpus en el análisis de textos literarios en español, un área aún escasa de análisis que adopten este tipo de enfoques. Esta escasez de estudios puede deberse, quizá, a las reticencias de una parte de la crítica a adoptar enfoques que partan de aspectos cuantitativos. Sin embargo, como hemos intentado demostrar, estas metodologías no pretenden reemplazar lo que se ha venido y se viene haciendo, sino complementar y reforzar algunas perspectivas de las que el análisis literario puede beneficiarse en gran medida.

\section{BIBLIOGRAFÍA CITADA}

ALARCos LloRACH, E. (1969): «Aditamento, adverbio y cuestiones conexas». Archivum, 19, págs. 301-329, (reimpr. en Estudios de gramática funcional del español. Madrid, Gredos, 1980, págs. 307-341).

ARRoyo DíEz, M.C. (2011): Aspectos espaciales y visuales en las primeras novelas contemporáneas Benito Pérez Galdós y su repercusión en la novela española actual. Tesis doctoral, Universidad de Valladolid.

BALOSSI, G. (2014): A Corpus Linguistic Approach to Literary Language and Characterization: Virginia Woolf's The Waves. Amsterdam, John Benjamins.

CALDAS-CoulthaRD, C.R. (1987): «Reported speech in written narrative texts». En Coulthard, M: Discussing Discourse. Birmingham, University of Birmingham, págs. 149-167.

CARBONERo, P. (1978): «Criterios para una caracterización funcional de los adverbios». Revista Española de Lingüística, 8, págs. 167-197. 
CASARES, J. (1992): Introducción a la lexicografía moderna. Madrid, CSIC.

Culpeper, J. (2001): Language and Characterisation. People in Plays and Other Texts. Harlow, Pearson Education.

- (2009): «Keyness: Words, parts-of-speech and semantic categories in the character-talk of Shakespeare's Romeo and Juliet». International Journal of Corpus Linguistics, 14.1, págs. 29-59.

Domínguez DE RodRíguez-PASQuÉs, P. (1970): «Morfología y sintaxis del adverbio en -mente». En Magis, C.H. (ed.): Actas del III Congreso Internacional de Hispanistas. México, El Colegio de México, págs. 293-303.

EGEA, E.R. (1979): Los adverbios terminados en -mente en el español contemporáneo. Bogotá, Instituto Caro y Cuervo.

FISCHER-STARCKE, B. (2010): Corpus Linguistics in Literary Analysis: Jane Austen and her Contemporaries. Londres, Continuum.

FuENTES RodRígueZ, C. (1987): "El adverbio de frase». Revista Española de Lingüística Aplicada, 3, págs. 55-74.

GARcía-PAGE, M. (1991): «El adverbio en -mente. Motivación contextual en formaciones léxicas anómalas». Anuario de Estudios Filológicos, 14, págs. 149-182.

— (1993): «Breves apuntes sobre el adverbio en -mente». Verba, 20, págs. 311340.

HAKEMUldER, F. (2016): «Empirical Stylistics». En Sotirova, V. (ed.): The Bloomsbury Companion to Stylistics. Londres-Nueva York, Bloomsbury, págs. 189207.

HallebeEK, J. (1985): «El adverbio. Bosquejo de una posible morfosintaxis del elemento adverbial en español». Dicenda, 4, págs. 35-56.

HaLlidAY, M.A.K. (2004): An Introduction to Functional Grammar. Londres, Arnold.

HARrison, C. (2017): Cognitive Grammar in Contemporary Fiction. Ámsterdam, John Benjamins.

Hernández Alonso, C. (1974): «El adverbio». Thesaurus. Boletín del Instituto Caro y Cuervo, 29, págs. 48-67.

KorTE, B. (1997): Body Language in Literature. Toronto, University of Toronto Press.

Kovacci, O. (1980): «Sobre los adverbios oracionales». Boletín de Filología de la Universidad de Chile, 31.2, págs. 519-535.

LAKOFF, G. (1970): «Adverbios y operadores modales». En Sánchez de Zavala, V. (ed.): Semántica y sintaxis en la lingüística transformatoria. II. Algunos temas y planteamientos nuevos. Madrid, Alianza, págs. 319-336. 
LAmbert, M. (1981): Dickens and the Suspended Quotation. New Haven, Yale University Press.

LÁZARo CARreter, F. (1953): Diccionario de términos filológicos. Madrid, Gredos. MAHLberg, M. (2013): Corpus Stylistics and Dickens's Fiction. Londres, Routledge.

- (2014): «Corpus stylistics». En Burke, M. (ed): The Routledge Handbook of Stylistics. Londres, Routledge, págs. 387-392.

MAhlBerg, M. y SMith, C. (2012): «Dickens, the suspended quotation and the corpus». Language and Literature, 21.1, págs. 51-65.

Mahlberg, M., Smith, C. y PReston, S. (2013): «Phrases in literary contexts: Patterns and distributions of suspensions in Dickens's novels». International Journal of Corpus Linguistics, 18.1, págs. 35-56.

McInTYRE, D. y Walker, B. (2019): Corpus Stylistics: Theory and Practice. Edimburgo, Edinburgh University Press.

Montoro, R. (2012): Chick Lit: The Stylistics of Cappuccino Fiction. Londres, Continuum.

Moreno DE AlBA, J.G. (1988): "Sobre la definición y clasificación del adverbio». Anuario de Letras: Lingüística y Filología, 26, págs. 31-66.

Nieto Caballero, G. (2018): «Metodologías de corpus en el análisis de textos literarios en lengua española: el ejemplo de Pérez Galdós». Estudios Humanísticos. Filología, 40, págs. 373-391.

Pattison, W.T. (1954): Benito Pérez Galdós and the creative process. Minneapolis, University of Minnesota Press.

RuANO SAN SEGUNDO, P. (2016): «A corpus-stylistic approach to Dickens' use of speech verbs: Beyond mere reporting». Language and Literature, 25.2, págs. 1-15.

ScotT, M. (2013): WordSmith Tools Manual. Version 6.0. Liverpool, Lexical Analysis Software.

Stockwell, P. (2009): Texture: A Cognitive Aesthetics of Reading. Edimburgo, Edinburgh University Press.

STUBBS, M. (2005): "Conrad in the computer: Examples of quantitative stylistics methods». Language and Literature, 14.1, págs. 5-24.

VERA LUJÁN, A. (1979): «La problemática gramatical/funcional en una tipología categorial. El adverbio en espafiol». Lexis, 3, págs. 171-194.

WEST, D. (2016): "Cognitive Stylistics». En Sotirova, V. (ed.): The Bloomsbury Companion to Stylistics. Londres, Bloomsbury, págs. 109-121. 


\section{APÉNDICES}

APÉNDICE 1: TEXTOS INCLUIDOS EN CORBPG

\begin{tabular}{|c|c|c|}
\hline NOVELA & AÑo & PALABRAS \\
\hline \multicolumn{3}{|l|}{ Episodios Nacionales. Primera serie } \\
\hline Trafalgar & 1873 & 51.453 \\
\hline La Corte de Carlos IV & 1873 & 139.656 \\
\hline El 19 de Marzo y el 2 de Mayo & 1873 & 136.403 \\
\hline Bailén & 1873 & 63.819 \\
\hline Napoleón en Chamartín & 1874 & 153.971 \\
\hline Zaragoza & 1874 & 133.010 \\
\hline Gerona & 1874 & 58.453 \\
\hline Cádiz & 1874 & 76.724 \\
\hline Juan Martín El Empecinado & 1874 & 63.757 \\
\hline La Batalla de los Arapiles & 1875 & 90.064 \\
\hline \multicolumn{3}{|l|}{ Episodios Nacionales. Segunda serie } \\
\hline El equipaje del Rey José & 1875 & 61.043 \\
\hline Memorias de un cortesano de 1815 & 1875 & 51.324 \\
\hline La Segunda Casaca & 1876 & 65.663 \\
\hline El Grande Oriente & 1876 & 64.034 \\
\hline 7 de Julio & 1876 & 51.624 \\
\hline Los Cien Mil Hijos de San Luis & 1877 & 56.331 \\
\hline El Terror de 1824 & 1877 & 67.105 \\
\hline Un voluntario realista & 1878 & 66.867 \\
\hline Los Apostólicos & 1879 & 77.113 \\
\hline Un faccioso más y algunos frailes menos & 1879 & 79.761 \\
\hline \multicolumn{3}{|l|}{ Episodios Nacionales. Tercera serie } \\
\hline Zumalacárregui & 1898 & 71.013 \\
\hline Mendizábal & 1898 & 83.240 \\
\hline De Oñate a La Granja & 1898 & 80.346 \\
\hline Luchana & 1899 & 93.695 \\
\hline La campaña del Maestrazgo & 1899 & 73.299 \\
\hline La estafeta romántica & 1899 & 67.799 \\
\hline Vergara & 1899 & 77.776 \\
\hline
\end{tabular}




\begin{tabular}{|c|c|c|}
\hline Montes de Oca & 1900 & 61.666 \\
\hline Los Ayacuchos & 1900 & 75.899 \\
\hline Bodas reales & 1900 & 74.812 \\
\hline \multicolumn{3}{|l|}{ Episodios Nacionales. Cuarta serie } \\
\hline Las tormentas del 48 & 1902 & 73.726 \\
\hline Narváez & 1902 & 82.797 \\
\hline Los duendes de la camarilla & 1903 & 72.494 \\
\hline La Revolución de Julio & 1903-04 & 77.393 \\
\hline O’Donnell & 1904 & 78.986 \\
\hline Aita Tettauen & 1904-05 & 78.507 \\
\hline Carlos VI en la Rápita & 1905 & 72.412 \\
\hline La vuelta al mundo en la Numancia & 1906 & 70.956 \\
\hline Prim & 1906 & 79.099 \\
\hline La de los tristes destinos & 1907 & 87.885 \\
\hline \multicolumn{3}{|l|}{ Episodios Nacionales. Quinta serie } \\
\hline España sin Rey & 1907-08 & 78.784 \\
\hline España trágica & 1909 & 76.574 \\
\hline Amadeo I & 1910 & 73.434 \\
\hline La Primera República & 1911 & 68.228 \\
\hline De Cartago a Sagunto & 1911 & 68.285 \\
\hline Cánovas & 1912 & 68.306 \\
\hline \multicolumn{3}{|l|}{ Novelas serie primera época } \\
\hline La sombra; Celín; Tropiquillos; Theros & 1870 & 48.343 \\
\hline El audaz: historia de un radical de antaño & 1871 & 119.174 \\
\hline Doña Perfecta: novela original & 1876 & 65.486 \\
\hline Gloria & $1876-77$ & 127.697 \\
\hline Marianela & 1878 & 51.234 \\
\hline La familia de León Roch & 1878 & 145.720 \\
\hline \multicolumn{3}{|l|}{ Novelas serie contemporánea } \\
\hline La desheredada & 1881 & 141.130 \\
\hline El amigo Manso & 1882 & 90.703 \\
\hline El doctor Centeno & 1883 & 113.949 \\
\hline Tormento & 1884 & 83.370 \\
\hline La de Bringas & 1884 & 71.862 \\
\hline
\end{tabular}




\begin{tabular}{|l|c|r|}
\hline Lo prohibido & $1884-85$ & 172.610 \\
\hline Fortunata y Jacinta: (dos historias de casadas) & $1886-87$ & 379.607 \\
\hline Miau & 1888 & 97.628 \\
\hline La incógnita & 1889 & 75.503 \\
\hline Realidad: novela en cinco jornadas & 1889 & 76.091 \\
\hline $\begin{array}{l}\text { Torquemada en la hoguera; El artículo de fondo; La } \\
\text { mula y el buey, La pluma en el viento; La conjura- } \\
\text { ción de las palabras; Un tribunal literario; La prin- } \\
\text { cesa y el granuja; Junio }\end{array}$ & 1889 & 62.658 \\
\hline Torquemada en la Cruz & & \\
\hline Torquemada en el Purgatorio & 1893 & 58.302 \\
\hline Torquemada y San Pedro & 1894 & 67.625 \\
\hline Ángel Guerra & 1895 & 61.367 \\
\hline Tristana & $1890-91$ & 249.534 \\
\hline La loca de la casa: comedia en cuatro actos & 1892 & 52.808 \\
\hline Nazarín & 1892 & 36.527 \\
\hline Halma & 1895 & 65.019 \\
\hline Misericordia & 1895 & 76.162 \\
\hline El abuelo: (novela en cinco jornadas) & 1897 & 84.131 \\
\hline \multicolumn{2}{|c|}{ Total PALABRAs } & 68.161 \\
\hline
\end{tabular}

APÉNDICE 2: TEXTOS INCLUIDOS EN CORXIX

\begin{tabular}{|c|l|c|r|}
\hline AUTOR & \multicolumn{1}{|c|}{ NOVELA } & AÑO & PALABRAS \\
\hline \multirow{4}{*}{ Alarcón } & El final de Norma & 1855 & 41.892 \\
\cline { 2 - 4 } & El sombrero de tres picos & 1874 & 24.909 \\
\cline { 2 - 4 } & El escándalo & 1875 & 78.583 \\
\cline { 2 - 4 } & El Capitán Veneno & 1881 & 40.655 \\
\hline \multirow{4}{*}{$\begin{array}{c}\text { Blasco } \\
\text { Ibáñez }\end{array}$} & Arroz y tartana & 1894 & 91.328 \\
\cline { 2 - 4 } & La barraca & 1898 & 54.575 \\
\cline { 2 - 4 } & Entre naranjos & 1900 & 80.873 \\
\cline { 2 - 4 } & Cañas y barro & 1902 & 4.913 \\
\cline { 2 - 4 } & La catedral & 1903 & 98.430 \\
\cline { 2 - 4 } & El intruso & 1904 & 96.969 \\
\hline
\end{tabular}




\begin{tabular}{|c|c|c|c|}
\hline & La horda & 1905 & 101.600 \\
\hline & Los argonautas & 1914 & 205.215 \\
\hline & Los cuatro jinetes del Apocalipsis & 1916 & 132.439 \\
\hline & Los enemigos de la mujer & 1919 & 163.461 \\
\hline & El paraíso de las mujeres & 1922 & 90.540 \\
\hline & El fantasma de las alas de oro & 1930 & 70.788 \\
\hline \multirow{2}{*}{ Clarín } & La Regenta & $1884-85$ & 309.796 \\
\hline & Su único hijo & 1890 & 89.969 \\
\hline \multirow{7}{*}{ Coloma } & La almohadita del niño Jesús & 1887 & 21.753 \\
\hline & Pequeñeces & $1890-91$ & 20.246 \\
\hline & Cuentos para niños & 1890 & 17.095 \\
\hline & La reina mártir & 1898 & 77.077 \\
\hline & Jeromín & 1902 & 146.177 \\
\hline & Colección de lecturas recreativas & 1902 & 216.807 \\
\hline & Por un piojo...: cuadro de costumbres & 1912 & 26.915 \\
\hline \multirow{4}{*}{$\begin{array}{l}\text { Palacio } \\
\text { Valdés }\end{array}$} & El idilio de un enfermo & 1884 & 58.113 \\
\hline & La aldea perdida & 1903 & 107.153 \\
\hline & La novela de un novelista & 1921 & 103.561 \\
\hline & Sinfonía pastoral & 1931 & 107.154 \\
\hline \multirow{14}{*}{$\begin{array}{l}\text { Pardo } \\
\text { Bazán }\end{array}$} & $\begin{array}{l}\text { Pascual López: autobiografía de un estu- } \\
\text { diante de Medicina }\end{array}$ & 1879 & 62.435 \\
\hline & Un viaje de novios & 1881 & 69.014 \\
\hline & La tribuna & 1883 & 65.160 \\
\hline & El Cisne de Vilamorta & 1885 & 53.123 \\
\hline & Los pazos de Ulloa & $1886-87$ & 84.000 \\
\hline & La madre naturaleza & 1887 & 90.463 \\
\hline & Insolación (Historia amorosa) & 1889 & 42.849 \\
\hline & Una cristiana & 1890 & 61.242 \\
\hline & La prueba & 1890 & 65.040 \\
\hline & La piedra angular & 1891 & 65.040 \\
\hline & Doña Milagros & 1894 & 67.826 \\
\hline & Memorias de un solterón & 1896 & 58.184 \\
\hline & El tesoro de Gastón & 1897 & 33.367 \\
\hline & El saludo de las brujas & 1899 & 60.078 \\
\hline
\end{tabular}




\begin{tabular}{|c|c|c|c|}
\hline & El niño de Guzmán & 1900 & 31.830 \\
\hline & Misterio & 1902 & 107.472 \\
\hline & La quimera & 1905 & 155.430 \\
\hline & La sirena negra & 1908 & 40.553 \\
\hline & Dulce dueño & 1911 & 66.499 \\
\hline & La última fada & 1916 & 15.011 \\
\hline \multirow{19}{*}{ Pereda } & Escenas montañesas & 1864 & 89.339 \\
\hline & Tipos y paisajes & 1871 & 100.753 \\
\hline & Don Gonzalo González de la Gonzalera & 1878 & 88.297 \\
\hline & El buey suelto... & 1878 & 72.537 \\
\hline & De tal palo tal astilla & 1880 & 85.974 \\
\hline & El sabor de la tierruca & 1882 & 81.756 \\
\hline & Pedro Sánchez & 1883 & 110.193 \\
\hline & Sotileza & 1885 & 117.441 \\
\hline & Esbozos y rasguños & 1888 & 70.323 \\
\hline & La Montálvez & 1888 & 106.115 \\
\hline & La mujer del César & 1888 & 25.179 \\
\hline & Los hombres de pro & 1888 & 45.060 \\
\hline & Tipos trashumantes & 1888 & 29.631 \\
\hline & Nubes de estío & 1891 & 121.738 \\
\hline & Al primer vuelo: Idilio vulgar. & 1891 & 100.914 \\
\hline & Oros son triunfos & 1894 & 27.233 \\
\hline & Peñas arriba & 1895 & 134.976 \\
\hline & Pachín González & 1896 & 60.309 \\
\hline & Escritos de juventud & $1954^{13}$ & 82.726 \\
\hline \multirow{6}{*}{ Valera } & Pepita Jiménez & 1874 & 56.575 \\
\hline & Las ilusiones del doctor Faustino & 1875 & 119.890 \\
\hline & Pasarse de listo & 1878 & 51.851 \\
\hline & Morsamor & 1899 & 84.574 \\
\hline & Juanita la Larga & 1895 & 73.329 \\
\hline & Genio y figura & 1897 & 53.358 \\
\hline
\end{tabular}

${ }^{13}$ Aunque su escritura fue anterior (entre 1858 y 1879), la edición que manejamos es de ese año. 


\begin{tabular}{|c|c|c|}
\hline Elisa la malagueña & $1964^{14}$ & 11.844 \\
\hline Leyendas del antiguo oriente & 1964 & 35.652 \\
\hline Mariquita y Antonio & 1964 & 50.824 \\
\hline Doña Luz & 1879 & 59.517 \\
\hline El comendador Mendoza & 1876 & 63.355 \\
\hline \multicolumn{2}{|l|}{ TOTAL PALABRAS } & 6.188 .478 \\
\hline
\end{tabular}

Guadalupe NiETo CABALLERO

Universidad de Extremadura gnieto@unex.es 0000-0001-5166-7057

${ }^{14}$ Elisa la malagueña, Leyendas del antiguo oriente y Mariquita y Antonio fueron publicadas antes de la fecha indicada: 1896, 1870 y 1861, respectivamente. 
